\title{
Effect of PEDOT:PSS Layer and ITO Ozonization in Arylenevinylene-co-Pyrrolenevinylene (AVPV) Based Solar Cell Devices
}

\author{
Ankur Solanki ${ }^{1,2}$, S. Sundar Kumar Iyer $^{2,3}$, Ashish Garg ${ }^{1,2}$ \\ ${ }^{1}$ Department of Materials Science \& Engineering, Indian Institute of Technology, Kanpur, India; ${ }^{2}$ Samtel Center for Display Tech- \\ nologies, Indian Institute of Technology, Kanpur, India; ${ }^{3}$ Departmental of Electrical Engineering, Indian Institute of Technology, \\ Kanpur, India. \\ E-mail: solanki@iitk.ac.in
}

Received September $26^{\text {th }}, 2011$; revised November $1^{\text {st }}, 2011$; accepted November $17^{\text {th }}, 2011$.

\begin{abstract}
Arylenevinylene-co-pyrrolenevinylene (AVPV) is polymer oligomer system derived from arylbridged bispyrroles which has been explored for photovoltaic devices. In this paper, we show the dependence of the photovoltaic device parameters on the anode surface treatment in an organic single layer photovoltaic device based on AVPV as an electron donor. Since the total quantum efficiency includes the charge collection efficiency at the electrodes, experiments were carried out to vary the anode (ITO) characteristics, achieved by using ITO with or without ozonization and with or without PEDOT:PSS (Polyethylene dioxythiophene:Polystyrene sulphonic acid) layer. Devices fabricated on ITO anode (without ozonization and without PEDOT:PSS) exhibited the maximum current density $\left(J_{s c}=1.3 \mu \mathrm{A} \cdot \mathrm{cm}^{-2}\right)$ as compared to those devices where ITO was ozonized as well as had a PEDOT:PSS layer $\left(J_{s c}=0.1 \mu \mathrm{A} \cdot \mathrm{cm}^{-2}\right)$ measured under 1 sun illumination of AM 1.5 through a calibrated solar simulator.
\end{abstract}

Keywords: AVPV, Organic Polymers, Ozonization, PEDOT:PSS, Organic Solar Cells

\section{Introduction}

Photovoltaic solar cells based on organic compounds are promising candidates for solar energy conversion. They have the potential for cost effectiveness, mechanical flexibility and easy processing [1-3]. However, in order to compete with the inorganic thin film solar cells, power conversion efficiencies of more than $10 \%$ must be achieved. While current research efforts have led to the efficiencies above $8 \%$ [4] There is a lack of certainty on the maximum achievable efficiencies and concerns on the device reliability and environmental stability of the organic solar cells (OSC) $[5,6]$. Moreover, for large area solar cell devices, development of materials which are environment friendly and manufacturing processes which are ecologically favourable is required.

As reviewed by Gunes et al., [7] a variety of polymers have been used to fabricate the organic solar cells and the results are dependent on the energy band alignment of the polymers with respect to other layers in the device. The internal power conversion efficiency of organic solar cell is mainly a product of five factors viz., 1) absorption efficiency, 2) exciton diffusion efficiency, 3) exciton dissociation efficiency, 4) charge transport efficiency and $5)$ charge collection efficiency $[8,9]$. It can be easily estimated that the polymers having low band gap $(<2 \mathrm{eV})$ absorb the light in the visible range $(300 \mathrm{~nm}-700 \mathrm{~nm})$ of solar spectrum. Since the first report of low band gap conjugated polymer, polyisothianaphthene (PITN) [10], much work has been carried out to prepare polymers with low band gap energy $[11,12]$. Low band gap conjugated polymers may have higher conductivity (carrier mobility) and therefore offer potential applications in transistors, transparent conductors, non-linear optical devices and smart windows.

In addition, stability of the active layer and rapid degradation of properties has been a major concern for OSC devices especially under light illumination and simultaneous exposure to oxygen or water vapor and an understanding of these aspects is crucial for fabricating long lasting devices. Degradation of organic solar cells device can occur due to various mechanisms such as diffusion of oxygen and moisture resulting in active layer and electrode degradation as reviewed by Jorgensen et al. [13]. 
During last few years, researchers have designed and synthesized a large number of $\pi$-conjugated arylenevinylene-co-pyrrolenevinylene (AVPV) oligomer system derived from arylbridged bispyrroles [14]. Bispyrroles are fluorescent dyes and have been used as the building blocks for fabricating the low band gap polymeric systems. In our previous work, we introduced a derivative of AVPV and investigated its candidature for the solar cells in single layer device structure [15]. The structure of this molecule is shown in Figure 1. The presence of alternate single and double bond in structure facilitate the conduction of electrons in this polymer. The device performance of photovoltaic cell is characterized by the short-circuit current density $\left(J_{s c}\right)$, open-circuit voltage $\left(V_{o c}\right)$ and fill factor $(F F)$. For single layer device structure, maximum ideal $V_{o c}$ is limited by the work function difference of electrodes used as cathode and anode $[5,16,17]$. In this work, we have a systematic study on the effect of variations in anode surface characteristics on charge collection efficiency. The study shows that total internal efficiency in single layer organic solar cell is also limited by the work function of used electrodes and HOMO (highest occupied molecular orbital)_LUMO (lowest occupied molecular orbital) levels of polymer $[18,19]$.

\section{Experimental Details}

\section{Device Fabrication}

The OSC devices were fabricated using AVPV as an active layer. A schematic of the device structure before encapsulation is shown in Figure 2. Transparent indium tin oxide (ITO)-coated glass substrates were used as anode for this study. Sheet resistance of the ITO layers was $16 \Omega \cdot \mathrm{sq}^{-1}$. ITO typically has a transitivity of $80 \%-90 \%$ in the UV range of light [1]. The ITO was patterned and then subjected to ozonization for selective experiments. The workfunction of ITO was measured as $4.8 \mathrm{eV}$ prior to ozonization and $5.1 \mathrm{eV}$ after ozonization. These values are in good agreement with the reported data [20,21].

For a few devices, thin film of PEDOT:PSS was spin coated on top of an ITO-coated substrates at $3000 \mathrm{rpm}$ for $90 \mathrm{~s}$. This layer was dried in the nitrogen ambient at $110^{\circ} \mathrm{C}$ for $1 \mathrm{~h}$.

To study the effect of variation in the anode characteristics, four different device structures were fabricated i.e. substrates with and without ozonization and with and without PEDOT:PSS. Active layers were spin coated at $1000 \mathrm{rpm}$ from a solution containing AVPV polymer. Concentration of AVPV solution was $10 \mathrm{mg} \cdot \mathrm{cc}^{-1}$ in chlorobenzene. The active layer was dried in a vacuum drying chamber at $120^{\circ} \mathrm{C}$ for $1 \mathrm{~h}$. For optical absorption measurements, a single layer of AVPV was deposited on a bare glass substrate whose results are shown in Figure 3.

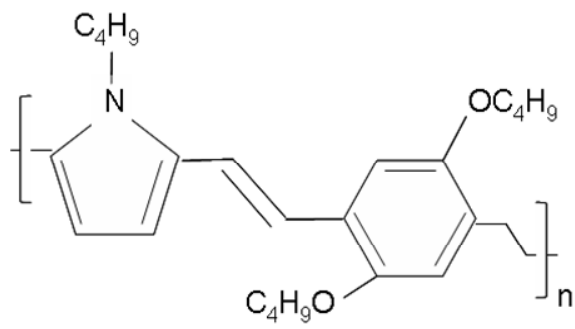

Figure 1. Molecular structure of Arylenevinylene-co-pyrrolenevinylene (AVPV).

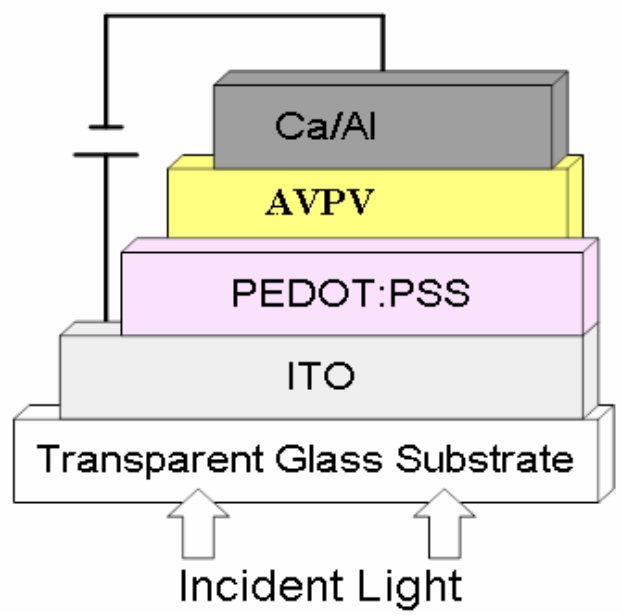

Figure 2. Schematic of the organic solar cell device structure.

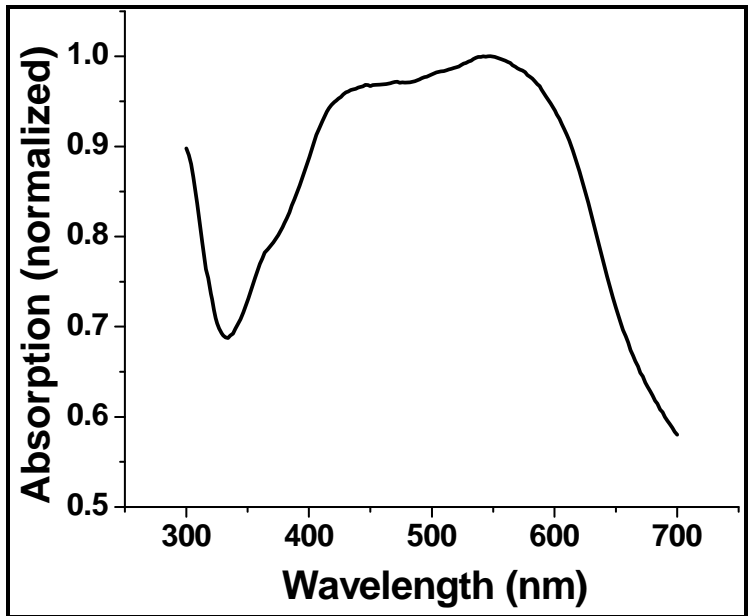

Figure 3. Normalized absorption spectrum of AVPV film in Chlorobenzene.

Top contacts of calcium-aluminium were evaporated on the top of the active layer in high vacuum of $10^{-6} \mathrm{mbar}$ to form the cathode. The final active device area for each cell was $0.2 \mathrm{~cm}^{2}$. Finally, the devices were encapsulated with epoxy in a nitrogen environment. 
The encapsulated devices were electrically characterized with a Keithley 4200 parameter analyser. Light characteristics were measured under 1 sun illumination of AM 1.5 through a calibrated solar simulator.

\section{Results and Discussion}

\subsection{Light Absorption}

To make a good quality organic solar cell, absorption spectrum of the organic material should match the Solar spectrum, as closely as possible. In addition, thickness of the active layer should be sufficient to achieve higher absorption. Generally, organic materials have higher absorption coefficients than inorganic semiconductors such as silicon [22]. Low band gap materials always tends absorb sunlight towards lower wavelength.

Figure 3 shows the normalized absorption spectrum taken on thin films of single layer AVPV, spun coated on a clean glass substrate. The figure shows that AVPV film has two broad peaks at $\sim 423 \mathrm{~nm}$ and $\sim 540 \mathrm{~nm}$ respecttively. The broad nature of these peaks suggests that AVPV absorb mainly in the visible region (between 400 $700 \mathrm{~nm}$ of the solar spectrum). This compares fairly well with the absorption spectra of well established polymer materials such as P3HT [23] and justifies the use of this polymer for the OSC devices as reported by us previously [15].

\subsection{Effect of Variations in the Anode Surface}

As a rule, the internal quantum efficiency $\left(\eta_{I Q E}\right)$ of OSCs depends on the collection efficiency of carriers at electrode. Collection of the charge carriers at the electrodes depends on the alignment of HOMO of the donor polymer and work function of the anode as well as alignment of LUMO of the polymer and work function of the cathode. As the difference between metal work function and LUMO or HOMO level reduces, it enhances the number of collected charge carriers at electrode and hence the internal quantum efficiency.

To the determine the effect of surface characteristics of anode, four different device structures of single layer AVPV devices, (A) ITO with ozonization and with PEDOT:PSS, (B) ITO with ionization and without PEDOT:PSS, (C) ITO without ionization and with PEDOT:PSS and (D) without ionization without PEDOT:PSS were fabricated. The energy band diagram of each of these device structures are shown in Figure 4. These device structures are fabricated to understand the change in the device performance after changes in the anode surface by means of ozonization, leading to change in the work function of ITO, as well as use of PEDOT:PSS.

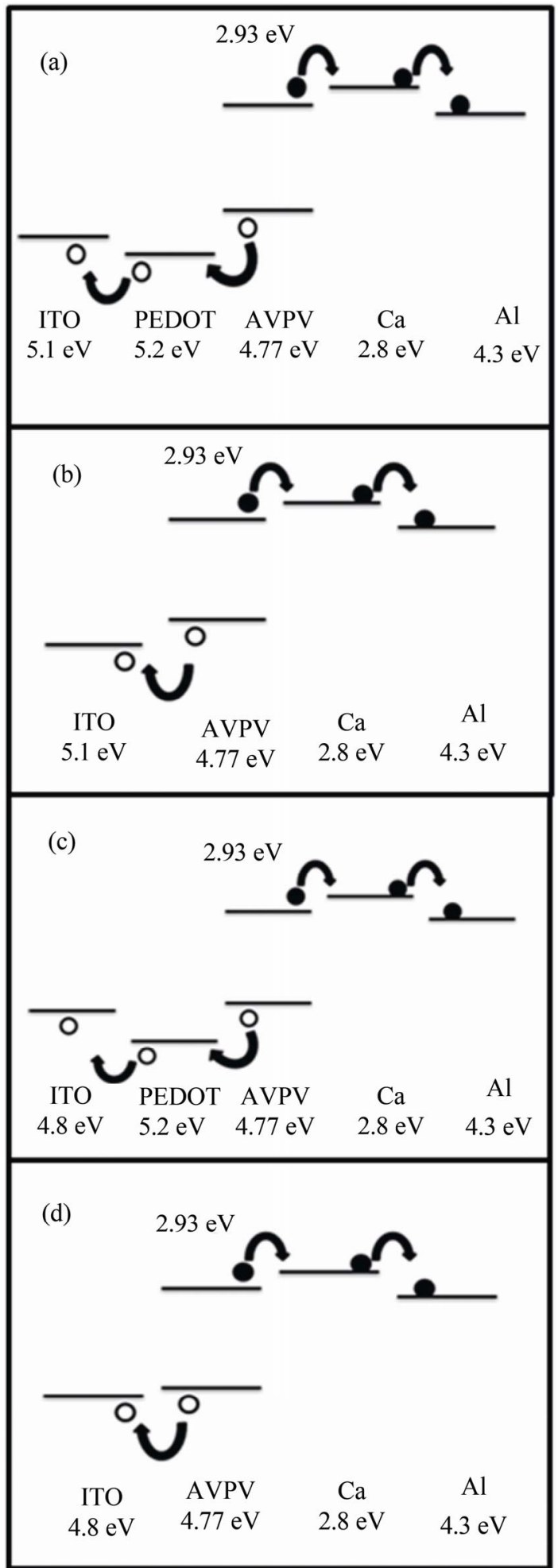

Figure 4. Energy band diagrams of single layer AVPV devices: (a) ITO with ionization and with PEDOT:PSS; (b) ITO with ionization and without PEDOT:PSS; (c) ITO without ionization and with PEDOT:PSS, and (d) ITO without ionization and without PEDOT:PSS. 
As our cyclic voltammetry measurements showed, the Highest Occupied Molecular Orbital (HOMO) level of AVPV lies at $4.77 \mathrm{eV}$ while PEDOT:PSS has a work function of $5.2 \mathrm{eV}$. Hence, as shown in the schematic band diagram in Figure 4(a), at the time of collection at anode, holes face a barrier of $0.43 \mathrm{eV}$. This indicated towards a reduction in the number of collected holes and thus may have an effect on the solar cell efficiency. In this context, ozonization of ITO also affects the collection because after ozonization as we found that ITO work functions shift from $4.8 \mathrm{eV}$ to $5.1 \mathrm{eV}$.

$J-V$ characteristics for all these devices measured under 1 sun illumination of AM 1.5 through a calibrated solar simulator and the results are shown in Figure 5. We find that the devices without ozonization and without PEDOT:PSS exhibit the highest short circuit current density $J_{s c}$, and devices with ozonization and with PEDOT:PSS exhibit the lowest $J_{s c}$ among these structures.

To perform a comparative study, the efficiency determining parameters are mentioned as a function of anode in Table 1. The table shows that the photovoltaic devices made with ionization with PEDOT:PSS are inferior in comparison to the devices from all other three type of devices. As shown in Figure 4(a), for the device structure (A), after the exciton dissociation, holes face a barrier of $0.43 \mathrm{eV}$ for the collection at anode as the accumulation should take place in the part of the device near anode.

This barrier reduces the hole collection at anode hampering the collection efficiency and hence total internal efficiency. Likewise, as the anode work function changes, barrier for collection of holes reduces from Figures 4(a)(d) and as a result the current density increases. The extracted device parameters for each of the devices (short circuit current density, $J_{s c}$, open circuit voltage, $V_{o c}$, Fill Factor, FF, series and shunt resistances, $R_{s}$ and $R_{s h}$ respectively) are shown in Table 1. In the following sections, we discuss these effects separately on each of the device parameter.

\subsubsection{Effect on $\boldsymbol{J}_{s c}$}

$J_{s c}$ is mainly controlled by efficiency of absorption of light, exciton dissociation, charge transport and charge collection. For all the four devices, absorption, dissociation and transportation are likely to be similar because of similar device thicknesses. The only variation is in the anode characteristics and band positions at the anode interface (Figure 4). The figure shows that as for the ITO anode without PEDOT:PSS and without ozonization (Device D), the ITO work function lies at $4.8 \mathrm{eV}$ which helps in aligning the HOMO level of AVPV with the ITO work function facilitating an easy transport of holes to the anode and hence increasing the $J_{s c}$. In contrast the devices which have a PEDOT:PSS layer, the holes face a larger barrier of $0.43 \mathrm{eV}$.

\subsubsection{Effect on Voc}

For a single layer OSC device, $V_{o c}$ is determined by the difference in the work function of both the electrodes [16]. This implies that the devices where ITO is ozonized and is further coated with PEDOT:PSS layer should show highest $V_{o c}$ (because of voltage drop at anode and active layer interface). But in these devices, charges also accumulate near the electrode and thus hampering the $V_{o c}$. In the device "D", no accumulation is likely so $V_{o c}$ was found to be maximum. Less $V_{o c}$ for device "B" having ITO with ozonization without PEDOT:PSS and device "C" without ozonization and with PEDOT:PSS. This appears to suggest that anode roughness may also have an effect on the $V_{o c}$ as PEDOT:PSS has a smoothening effect on ITO.

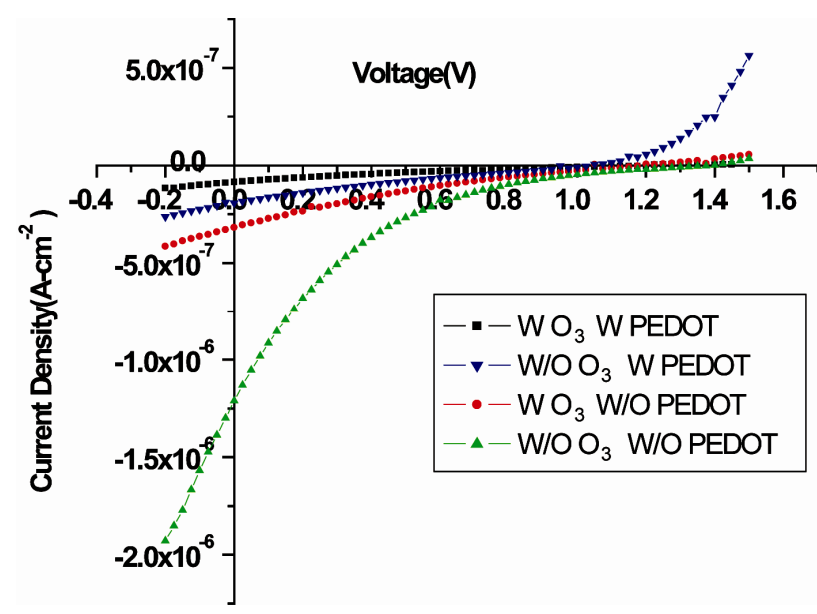

Figure 5. $J-V$ characteristics of single layer AVPV devices with anode variations under 1 Sun illumination.

Table 1. A comparison of device parameters for all four types of OSC devices with variations in anode characteristics.

\begin{tabular}{cccccc}
\hline Devices & $\begin{array}{c}J_{s c}\left(\mathrm{~A} \cdot \mathrm{cm}^{-2}\right) \\
\times 10^{-7}\end{array}$ & $V_{o c}(\mathrm{~V})$ & $\mathrm{FF}(\%)$ & $\begin{array}{c}R_{s} \\
\left(10^{6} \Omega \cdot \mathrm{cm}^{-2}\right)\end{array}$ & $\begin{array}{c}R_{s h} \\
\left(10^{6} \Omega \cdot \mathrm{cm}^{-2}\right)\end{array}$ \\
\hline $\mathrm{A}$ & 1.1 & 1.2 & 24 & 1.7 & 5.0 \\
$\mathrm{~B}$ & 2.1 & 1.05 & 19 & 1.8 & 2.8 \\
$\mathrm{C}$ & 4.3 & 1.05 & 18 & 1.1 & 1.4 \\
$\mathrm{D}$ & 13 & 1.4 & 9.1 & 0.6 & 0.2 \\
\hline
\end{tabular}




\subsubsection{Effect on FF}

Fill Factor (FF) is actually the filling factor (ratio of maximum power to the theoretical maximum power) determined by the fraction of the photo-generated charge carriers that actually reach the electrodes, when the builtin field is lowered toward the open circuit voltage. In fact, there is a competition between charge carrier recombination and charge transport. Highest shunt resistance for device " $\mathrm{D}$ " results in highest recombination and hence least FF [24].

\section{Conclusions}

In conclusion, we showed the effect of surface characteristics of anode in the single layer devices on the photovoltaic properties of the devices. Since the total quantum efficiency also includes the charge collection efficiency at the electrodes the energy level diagram of the device suggested that the alignment of ITO anode work function and HOMO level of donor is an important condition for better collection of holes under illumination. We found that the devices fabricated on ITO anode (without ozonization and without PEDOT:PSS) exhibited the maximum current density $\left(J_{S c}=1.3 \mu \mathrm{A} \cdot \mathrm{cm}^{-2}\right)$ as compared to those devices where ITO was ozonized as well as had a PEDOT:PSS layer $\left(J_{s c}=0.1 \mu \mathrm{A} \cdot \mathrm{cm}^{-2}\right)$. This is due to reduced barrier to the hole transport to the anode from the active layer when ITO is not ozonized and PEDOT:PSS layer is absent.

\section{Acknowledgements}

This work was financially supported by the Department of Science and Technology, Government of India, New Delhi.

\section{REFERENCES}

[1] G. A. Chamberlain, "Organic Solar Cells: A Review," Solar Cells, Vol. 8, No. 1, 1983, pp. 47-83. doi:10.1016/0379-6787(83)90039-X

[2] B. Gregg, "The Photoconversion Mechanism of Excitonic Solar Cells," Materials Research Bulletin, Vol. 30, No. 1, 2005, pp. 20-22. doi:10.1557/mrs2005.3

[3] H. Spanggaard and F. C. Krebs, "A Brief History of the Development of Organic and Polymeric Photovoltaics," Solar Energy Materials and Solar Cells, Vol. 83, No. 2-3, 2004, pp. 125-146. doi:10.1016/j.solmat.2004.02.021

[4] Z. He, C. Zhong, X. Huang, W. Y. Wong, H. Wu, L. Chen, S. Su and Y. Cao, "Simultaneous Enhancement of Open-Circuit Voltage, Short-Circuit Current Density, and Fill Factor in Polymer Solar Cells," Advanced Materials, Vol. 23, No. 40, 2011, pp. 4636-4643. doi:10.1002/adma.201103006

[5] H. Hoppe and N. S. Sariciftci, "Organic Solar Cells: An
Overview," Journal of Materials Research, Vol. 19, No. 7, 2004, pp. 1924-1945. doi:10.1557/JMR.2004.0252

[6] E. Soto, J. C. MacDonald, C. G. F. Cooper and W. G. McGimpsey, "A Non-Covalent Strategy for the Assembly of Supramolecular Photocurrent-Generating Systems," Journal of the American Chemical Society, Vol. 125, No. 10, 2003, pp. 2838-2839. doi:10.1021/ja0289548

[7] S. Gunes, H. Neugebauer and N. S. Sariciftci, "Conjugated Polymer-Based Organic Solar Cells," Chemistry Reviews, Vol. 107, No. 4, 2007, pp. 1324-1338. doi:10.1021/cr050149z

[8] P. W. M. Blom, V. D. Mihailetchi, L. J. A. Koster and D. E. Markov, "Device Physics of Polymer: Fullerene Bulk Heterojunction Solar Cells," Advanced Materials, Vol. 19, No. 12, 2007, pp. 1551-1566. doi:10.1002/adma.200601093

[9] W. U. Huynh, J. J. Dittmer and A. P. Alivisatos, "Hybrid Nanorod-Polymer Solar Cells," Science, Vol. 295, No. 5564, 2002, pp. 2425-2427. doi:10.1126/science.1069156

[10] S. A. Chen and C. C. Lee, "Processable Low Band Gap N-Conjugated Polymer, Poly (Isothianaphthene): Its Synthesis and Reaction Mechanism," Pure and Applied Chemistry, Vol. 67, No. 12, 1995, pp. 1983-1990. doi: $10.1351 / \mathrm{pac} 199567121983$

[11] E. Bundgaard and F. C. Krebs, "Low Band Gap Polymers for Organic Photovoltaics," Solar Energy Materials and Solar Cells, Vol. 91, No. 11, 2007, pp. 954-985. doi:10.1016/j.solmat.2007.01.015

[12] M. M. Wienk, M. P. Struijk and R. A. J. Janssen, "Low Band Gap Polymer Bulk Heterojunction Solar Cells," Chemical Physics Letters, Vol. 422, No. 4-6, 2006, pp. 488-491. doi:10.1016/j.cplett.2006.03.027

[13] M. Jorgensen, K. Norrman and F. C. Krebs, "Stability/Degradation of Polymer Solar Cells," Solar Energy Materials \& Solar Cells, Vol. 92, No. 7, 2008, pp. 686714. doi:10.1016/j.solmat.2008.01.005

[14] A. K. Biswas, Ashish, A. K. Tripathi, Y. N. Mohapatra and A. Ajayaghosh, "Synthesis, Photophysical, and Electroluminescent Properties of Arylenevinylenes-Copyrrolenevinylenes Derived from Divinylaryl Bridged Bispyrroles," Macromolecules, Vol. 40, No. 8, 2007, pp. 26572665. doi: $10.1021 / \mathrm{ma} 070116 \mathrm{~g}$

[15] A. Solanki, A. Gupta, S. S. K. Iyer and A. Garg, "Photo Voltaic Effect in Arylenevinylene-co-Pyrrolenevi Nylene (AVPV)," Solar Energy Materials and Solar Cells, Vol. 93, No. 2, 2009, pp. 211-214. doi:10.1016/j.solmat.2008.09.029

[16] C. J. Brabec, A. Cravino, D. Meissner, N. S. Sariciftci, M. T. Rispens and L. Sanchez, "The Influence of Materials Work Function on the Open Circuit Voltage of Plastic Solar Cell," Thin Solid Films, Vol. 403-404, 2002, pp. 368-372. doi:10.1016/S0040-6090(01)01586-3

[17] M. Ramsdale, J. A. Barker, A. C. Arias, J. D. MacKenzie and R. H. Friend, "The Origin of the Open-Circuit Voltage in Polyfluorene-Based Photovoltaic Devices," Journal of Applied Physics, Vol. 92, No. 8, 2002, pp. 4266- 
4270. doi:10.1063/1.1506385

[18] A. Geiser, B. Fan, H. Benmansour, F. Castro and J. Heier, "Poly(3-Hexylthi Ophene)/C60 Hetero Junction Solar Cells: Implication of Morphology on Performance and Ambipolar Charge Collection," Solar Energy Materials and Solar Cells, Vol. 92, No. 4, 2008, pp. 464-473. doi:10.1016/j.solmat.2007.11.001

[19] S. W. Oh, H. W. Rhee, C. Lee and Y. C. Kim, "The Photo-Voltaic Effect of the p-n Heterojunction Organic Photo-Voltaic Device Using a Nano Template Method," Current Applied Physics, Vol. 5, No. 1, 2005, pp. 55-58. doi:10.1016/j.cap.2003.11.079

[20] N. Srivastava, "Circuit Modeling of P3HT:PCBM Based on Blend Solar Cellsand Experimental Study of Effect of PEDOT:PSS Layer on These Solar Cells," M.Tech Dissertation, Departmental of Electrical Engineering, Indian Institute of Technology, Kanpur, 2008.
[21] Z. R. Hong, C. J. Liang and X. Y. Sun, "Characterization of Organic Photovoltaic Devices with Indium-Tin-Oxide Anode Treated by Plasma in Various Gases," Journal of Applied Physics, Vol. 100, No. 9, 2006, Article ID: 093711. doi:10.1063/1.2372574

[22] B. Gregg, "The Photoconversion Mechanism of Excitonic Solar Cells," MRS Bulletin, Vol. 30, 2005, pp. 20-22. doi: $10.1557 / \mathrm{mrs} 2005.3$

[23] V. Shrotriya, J. Ouyang, R. J. Tseng, G. Li and Y. Yang, "Absorption Spectra Modification in Poly(3-Hexylthiophene):Methanofullerene Blend Thin Films," Chemical Physics Letters, Vol. 411, No. 1-3, 2005, pp. 138-143. doi:10.1016/j.cplett.2005.06.027

[24] D. Gupta, M. Bag and K. S. Narayan, "Correlating Reduced Fill Factor in Polymer Solar Cells to Contact Effects," Applied Physics Letters, Vol. 92, No. 9, 2008, Article ID: 093301. 